Discussion C21 attenuates clinical and histological signs in established CIA. The expansion of the relative number of CD4+Treg cells might be responsible for the observed antiinflammatory effect induced by C21. Nevertheless, further experiments are required to characterise the underlying mechanisms. As C21 does not seem to exhibit severe side-effects it might become an attractive candidate for arthritis therapy.

\title{
7 A NON-PEPTIDE AT2 RECEPTOR AGONIST ATTENUATES CLINICAL AND HISTOLOGICAL SIGNS IN MURINE COLLAGEN-INDUCED ARTHRITIS
}

Bettina Sehnert, ${ }^{1}$ Georg Schett, ${ }^{2}$ Reinhard E Voll, ${ }^{1,3}$ Ulrike M Steckelings ${ }^{4}{ }^{1}$ Center of Chronic Immunodeficiency, University Hospital Freiburg, Freiburg, Germany; ${ }^{2}$ Department of Rheumatology and Clinical Immunology, University Hospital Erlangen, University of Erlangen-Nürnberg, Erlangen, Germany; ${ }^{3}$ Department of Rheumatology and Clinical Immunology, University Hospital Freiburg, Freiburg, Germany; ${ }^{4}$ CharitéUniversity Medicine Berlin, Center for Cardiovascular Research, Berlin, Germany

10.1136/annrheumdis-2011-201239.7

Background and objectives The renin-angiotensin system is known to play a critical role in inflammation, fibrosis, and end-organ damage. Nevertheless, anti-inflammatory, antifibrotic and antiapoptotic features regulated by angiotensin II type 2 receptor (At2R) signalling are increasingly discussed. Compound 21 (C21) is the first non-peptide AT2 receptor agonist that stimulates selectively the AT2 receptor without affecting the AT1 receptor. This study was designed to examine the impact of C21 on collagen-induced arthritis (CIA) to investigate if AT2 receptor agonists might represent promising candidates for the treatment of inflammatory and autoimmune diseases.

Materials and methods Daily treatment with C21 was started 20 days after CIA induction prior to the onset of clinical symptoms by intraperitoneally injections. Etanercept was used as a positive control. Mice were monitored daily starting from day 20 after immunisation for signs of arthritis in the hind and front paws by two independent observers. The clinical severity of arthritis was quantified according to a graded scale (0-4) for each paw and expressed as mean score of all four paws. After sacrificing the mice at day 45 the hind paws were fixed in $4 \%$ formalin then decalcified with EDTA and embedded in paraffin for histological analysis. H\&E, toluidine blue and tartrate-resistant acid phosphatase stained sections were graded on a scale from $0-3$. TH1 cytokines and Tregs were analysed by a multiplex assay using flow cytometry.

Results C21 administered intraperitonially starting 20 days after CIA induction suppressed clinical symptoms on day 45 $(p<0.05)$ compared to the PBS group. The effect of C21 on joint swelling was comparable to Etanercept which the authors used as a positive control. Analysing the cumulative incidence, a slower development of the disease was detected in C21 treated mice. Histological scores of the hind paws showed lower numbers of inflammatory cells $(p<0.01)$ and milder cartilage degradation $(p<0.01)$, whereas no significant difference was detectable on bone destruction. Antimouse CII IgG2a levels did not differ significantly between the groups. A trend was observed towards a decrease in IL- $1 \alpha$ concentrations after C21 treatment. Furthermore, C21 treated CIA-mice showed an increased number of CD4+Foxp3+cells. 